\title{
THE ISOLATION AND CHARACTERS OF L-FORMS AND \\ REVERSIONS OF BACILLUS LICHENIFORMIS VAR. ENDOPARASITICUS (BENEDEK) ASSOCIATED WITH THE ERYTHROCYTES OF CLINICALLY NORMAL PERSONS
}

\author{
K. A. Bisset AND Roberta Bartlett \\ Department of Bacteriology, University of Birmingham, Birmingham B15 $2 T J$
}

Plate XXXV

MANY isolations have been recorded of Gram-positive bacteria from the blood and body fluids of human subjects, in disorders normally regarded as noninfective, or from normal persons; and frequent claims have been made of a positive relationship between these bacteria and conditions with a postulated immunological element, such as cancer and arthritis. One organism has especially often been incriminated: Benedek (1955) compiled evidence to prove that a bacterium, " Bacillus endoparasiticus", often occurred in blood and body fluids in association with arthritis; he described the organism in rather vague terms, although the characteristic, licheniform colony is clearly seen in his illustrations. Livingston and Alexander-Jackson (1970), who were mainly concerned with strains derived from neoplasms, including human cancers, fully recorded the organism's unusual morphological characters and suggested that it belonged to the Actinomycetales. These workers proposed a new genus Cryptocides, but acknowledged the organism's possession of a Bacillus-type endospore and this would seem to invalidate the classification. They also illustrated the typical, fimbriate, licheniform colony of the mature isolate.

Pease (1969) and Markham and Myers (1976), in studies concerned with arthritis, showed that the bacillus can frequently be isolated in the first stages of primary culture as a spheroplast that may take weeks to produce visible growth. The spheroplast usually reverts to a small "diphtheroid" bacterium, and this is most frequently the stage to be observed and isolated (see Pease, 1967a). The spheroplasts and the early small bacteria are readily recognisable because they are acid-fast, and this stage has been noticed especially by workers studying the aetiology of cancer (Alexander-Jackson, 1954). On subculture, or in prolonged primary culture, the diphtheroids increase in size and cease to be acid-fast. Thereafter, they usually revert further, to a Gram-positive bacillus that may form spores (Livingston and AlexanderJackson, 1970; Pease, 1974). The large diphtheroids and Gram-positive bacilli of the nonsporing variety have been reported most often by workers studying rheumatoid arthritis (e.g., Duthie et al., 1967). Pease(1967a and 1969)originally identified the diphtheroid as a member of the genus Listeria, to which it is serologically related, but subsequently identified it as an intermediate L-form of a species of Bacillus resembling Bacillus licheniformis (Pease, Nicholls and Stuart, 1972; Pease, 1974) confirming the view of Roberts (1964). Provisionally it may be called Bacillus licheniformis var. endoparasiticus (Benedek) hereinafter referred to as BLE. It should be noted that $B$. licheniformis is a relatively small sporing bacillus, approximately $0.7 \mu \mathrm{m}$ in width, so that the progression in morphology does not represent any great alteration

Received 12 Jan. 1978; accepted 7 Feb. 1978.

J. MED. MICROBIOL.-VOL. 11 (1978) 
in appearance as shown by Gram's stain or in size from that of the larger diphtheroid rods; indeed, any small non-sporing, Gram-positive rod is liable to be classed as a diphtheroid in routine bacteriology. The colony of $B$. licheniformis is complex and comprises phase variants comparable with those of Proteus spp. (Bisset and Street, 1973), and this, in conjunction with the effects of the L-cycle, accounts for some of the morphological variability described.

Although both Benedek (1955) and Pease (1974) associated the bacillus with arthritis and a variety of diseases, they suggested that it may be universal in human beings, and not a conventional pathogen. Villequez (1965), who isolated such a bacillus from cancer patients, found it also in the blood of $20 \%$ of normal persons. However, a proper healthy-control series examined by the same methods as those used for isolations from pathological material has not previously been available, and is presented here. A preliminary letter, discussing part of this work in relation to isolations claimed to be relevant to the aetiology of arthritis, has been published (Bisset, 1977).

Investigations of this problem present unusual difficulties. Work with putatively uninfected experimental animals, including control blood sampling and antigenic analysis, is prejudiced because most vertebrates, like human beings, appear to be liable to harbour this organism or a related species; thus, in addition to introducing their own strains to the experiment, they may be immunologically tolerant or carry natural antibodies (Pease, $1965 a$ and 1967a). An identification of the L-form of $B$. licheniformis was, in fact, first made upon material from birds (Roberts, 1964).

Although they commonly occur as parasites and pathogens of man and other animals, sporing bacteria are automatically suspected to be contaminants when isolated (e.g., Marmion, 1976). Bacteriologists tend to avoid reporting their appearance in cultures, and this has led to the loss of many observations from the record; moreover, the dissimilar growth phases of BLE are liable to be identified as different types of micro-organism if they are not studied in culture over an extended period. Nevertheless, the peculiar characters and behaviour of this bacillus enable its identity to be recognised in the numerous descriptions that have appeared over many years in bacteriological literature.

\section{MATERIALS AND METHODS}

Sources of strains

Blood. One hundred samples of whole human blood, from healthy donors, were taken by courtesy of the West Midland Regional Blood Transfusion Service. The donor's skin was first disinfected by the application, on a fresh sterile cotton-wool swab held in forceps, of methylated spirit of soap followed after 3-5 min. by $0.5 \%$ chlorhexidine in $70 \%$ alcohol. Blood was taken for transfusion by means of new, disposable " taking sets" (Fenwal Ltd, Thetford, Norfolk), with a fresh set for each sample. These had been sterilised by autoclaving at $121^{\circ} \mathrm{C}$ for $45 \mathrm{~min}$., and each batch of manufacture was tested for sterility. Sets were transported in sterile metal containers before use, so that the sterility of the outside of the tubes was preserved. Each sample was taken at the conclusion of the bleeding, but while the apparatus was still attached to the donor's vein, by severing the tube with sterile scissors at a point where it had not been touched by any unsterile object. Two 10-ml samples were first taken for other purposes and a third, with careful sterile precautions, was taken in a sterile $25-\mathrm{ml}$ screw-capped bottle (McCartney bottle) containing $1 \mathrm{ml}$ of sterile acid-citratedextrose anticoagulant; each bottle and its contents had been autoclaved at $121^{\circ} \mathrm{C}$ for $20 \mathrm{~min}$. All bottles containing anticoagulant were provided by and prepared in this laboratory, and regularly tested for sterility.

On each occasion, sub-sampling was performed by means of a fresh sterile 1-ml pipette, with standard flaming procedures.

Samples of plasma from 55 healthy persons at the Institute of Physiology, University of Camerino, Italy, taken by new, disposable hypodermic syringes and transmitted to this 
laboratory in a refrigerated container, were provided by Professor G. Tedeschi. At a later date in this study, samples of blood taken at Ancona Hospital, Italy, from 100 patients suffering from a wide range of conditions not usually associated with blood infections, were inoculated into PPLO broth at Ancona, and the cultures and isolates were examined in this laboratory. These were also provided by Professor G. Tedeschi, the blood samples having been taken with new, disposable hypodermic syringes. All the Italian samples were taken after skin had been disinfected with alcoholic iodine.

Private culture collections. For comparison, the following strains, identified as BLE, were also examined: four provided by Dr Eleanor Alexander-Jackson, one by Dr Sakae Inoue, five by Dr Phyllis Pease, four by Dr D. H. Roberts, three by Dr Florence Siebert and ten by Professor G. Tedeschi.

\section{Culture methods}

Culture of whole blood. For initial blood and plasma cultures the media employed were PPLO broth (Difco, Detroit, USA), Brewer's medium (Difco) and the L-form medium described by Pease (1970), in 10-ml volumes in McCartney bottles. In each instance, $1 \mathrm{ml}$ of blood was added by pipette to $10 \mathrm{ml}$ of fluid medium and incubated at $37^{\circ} \mathrm{C}$, with the bottle cap firmly screwed down.

Culture of separated blood. Seventy samples of whole blood were separated by low-speed centrifugation, with sterile precautions. Deposit $(0.5 \mathrm{ml})$ and plasma $(1.0 \mathrm{ml})$ from each sample were used as culture inocula. Half the blood cell-deposit samples were inoculated in duplicate in PPLO broth and half in duplicate in L-form medium. Each plasma sample was inoculated into both PPLO broth and Brewer's medium. As these methods were similar to those of previous workers, it was hoped that valid comparisons with earlier studies would be possible.

Incubation. Cultures were incubated for periods of up to 25 months at $37^{\circ} \mathrm{C}$, with the exception of 30 Birmingham samples, and the samples of whole blood from Ancona; both of these sets were obtained relatively late in the experiment, and were incubated for $c .4$ months.

Subculture. Subcultures from all fluid cultures were made on Trypticase Soy Agar (Difco), PPLO agar (Difco) with $20 \%$ inactivated horse serum, and MacConkey's agar (Difco). Any organisms appearing in subculture were tested for their ability to grow in the fluid media listed. Subcultures were incubated in batches of eight plates, in closed, sterile plastic bags, for 3-4 weeks at $37^{\circ} \mathrm{C}$.

Examination. Fluid cultures were examined unopened for colour and turbidity at intervals of 2 days for the first 2 weeks and subsequently at weekly intervals. For the first 12 weeks, subcultures were made only when changes had been observed, and thereafter subcultures were made at monthly intervals from all initial cultures except the unopened controls (see below). Subcultures were recorded as positive only if they yielded a large number $(c .50-100)$ of identical colonies of BLE, distributed in accordance with the plating technique. In practice, they either yielded large numbers of colonies or failed to yield growth. When positive subcultures were obtained, the subculturing was immediately repeated from the original culture, and samples of at least $50 \%$ of the colonies on each plate were examined, first in situ by phase contrast microscopy (Bisset and Street, 1973) and then by Gram-stained smears; $c$. 10 colonies from each subculture were replated for purity before being subjected to physiological tests. Replating of large numbers of colonies was considered essential, because of the extremely protean morphology of BLE.

Induction of reversion. Reversion in purified subcultures was also induced by a method (Madoff, 1974) that entailed growth in the presence of metabolic products from other bacteria; staphylococci were used as Tedeschi (1977, personal communication) observed that they produced this effect with BLE.

\section{Control of cultural procedures}

Medium. A total of 40 uninoculated cultures of all media employed were incubated and subcultured for the entire period of the experiment, as negative controls. Duplicate cultures of 50 of the 100 whole-blood samples taken in Birmingham were made in the fluid media 
and left unopened throughout the period of the experiment, as controls for cultures that had yielded growth on subculture; a duplicate culture was opened only if it showed visible signs of growth, or if a culture from the same blood sample had produced BLE on subculture.

Collection of samples. Control of contamination at blood-taking sessions was effected by taking the blood samples at weekly intervals, in batches of approximately 10 , in one or other of two different buildings separated from each other by $c .1$ mile, both being $c .5$ miles from this laboratory. Each operator took only two or three samples, and four to six operators took samples on each occasion; $c .40$ different persons performed the collection. The possibility of aerial contamination in the rooms was tested by leaving four control bottles of sterile anticoagulant open beside the operator, throughout the period of each blood-taking session. The solutions were thereafter added to tubes of the test media and incubated exactly as for blood cultures. Sub-sampling was performed by at least three different operators in separate laboratories. Before these and similar procedures were carried out, the bench was cleaned and disinfected with a phenolic preparation (Hycolin; Wm Pearson Ltd, Hull).

Cultures and subcultures. Most initial cultures and subcultures were made in a laboratory in this department that was subject to very little contamination from air, dust, etc.; 40 control cultures were made from dust and air in this laboratory by exposing plates of Trypticase Soy Agar (Difco) and by culturing swabs of exposed surfaces on plates of the same medium; $20 \%$ of cultures were made in another laboratory, and four operators, all graduate bacteriologists of experience, made them at different times. Two incubators in separate rooms were used, and were regularly examined by swabbing and culture.

Simultaneously with any inoculation or subculture, an open plate of Trypticase Soy Agar was exposed on the bench immediately in front of the operator, at a distance of $c .50 \mathrm{~cm}$. The operator's skin and nose were also examined for organisms resembling BLE.

The skin and nose flora of samples representing $c .50 \%$ of the regular users of this laboratory are examined each year and, since the initiation of this study approximately 10 years ago, the relevant cultures have been examined by both visual and cultural methods for the presence of any organism resembling BLE.

On each occasion that the primary cultures were subcultured, at least six uninoculated bottles of each medium used, having first been incubated during the same period as the test cultures, were also subcultured.

Controls of inoculation, subculture and incubation procedures were made as follows. Horse blood $(1.0 \mathrm{ml})$ obtained from Tissue Culture Services Ltd, Slough, Bucks., and irradiated at 5 megarads, was added to each of 60 bottles of PPLO broth. This medium had proved to be very suitable for all phases of BLE. These bottles were subcultured at monthly intervals in parallel with blood samples from the usual sources. Deposit $(0.5 \mathrm{ml})$ of erythrocytes obtained from horse blood by centrifugation was added to each of a further 90 bottles, which were then autoclaved at $121^{\circ} \mathrm{C}$ for $15 \mathrm{~min}$; ; whole blood was not used, to obviate coagulation. The 90 bottles were subcultured at intervals of $c .3$ days. As the negativecontrol procedures were carried out after the original isolations had been made, a further series of blood cultures and subcultures was prepared, as a simultaneous positive control.

Effect on erythrocytes. Four strains of $B$. licheniformis, maintained in this laboratory as type cultures, and eight of BLE, were inoculated into bottles, each containing $10 \mathrm{ml}$ of PPLO broth with $1 \mathrm{ml}$ of fresh blood, to act as controls for the effect of the isolates upon erythrocytes. Both human blood, taken from the sources described above, and horse blood were used.

\section{Microscopical examination}

Slide preparations were made only from the cultures that had been opened for subculture, in order to avoid needless risk of contamination. They were examined by phase contrast and by Gram's stain. Growth appearing in subculture was examined by these means and also by (1) the Ziehl-Neelsen method modified by the use of $10 \%$ sulphuric acid in water or ethanol as a decolorising agent, and (2) the phosphomolybdic acid-methyl green method for cell walls. The latter method gives a good, general illustration of outline cellular morphology (Bisset, 1970). 


\section{Physiological tests}

Physiological tests were made on strains purified by repeated subculture and picking of colonies. The techniques were those described by Cowan and Steel (1974) except that longer periods of incubation were sometimes necessary. The tests included the following: peptonewater sugar fermentation; serum-water sugar fermentation; methyl red; Voges-Proskauer; production of urease; utilisation of citrate; hydrolysis of starch, casein and gelatin; oxidation and fermentation; oxidase; catalase; coagulase; reduction of nitrate. Spore resistance was tested at $80^{\circ} \mathrm{C}$ and $100^{\circ} \mathrm{C}$ in screw-capped bottles of Nutrient Broth (Difco).

Identification tests were also made independently by the Department of Microbiology, West of Scotland Agricultural College.

\section{RESULTS}

\section{Isolates}

Isolates from whole blood. Thirty-two isolates of BLE were obtained from 22 of 70 locally obtained samples of normal whole blood or blood cells, after incubation of primary cultures for periods ranging from less than 1 to $c .25$ months. In addition, five isolates were obtained from a further 30 similar samples, and 13 isolates were obtained from 100 cultures of patients' whole blood inoculated in Ancona; these isolates were obtained after relatively short periods of incubation ( $<5$ months), the isolation rates being similar in the two sets of samples. Subcultures developed within 1-5 days. L-form broth and PPLO broth were equally effective for isolation, but Brewer's medium was less so. These findings and those listed below are illustrated by the data given in table I.

Isolations from a particular culture could usually but not invariably be repeated at a later date, apparently because the reverting L-forms tended to die out readily in primary and later subcultures if they had failed to revert completely at once. Taking account of the different media and different types of inoculum, it will be apparent that each sample was cultured six times in parallel; in addition, each was cultured in duplicate in one of the major media. Of the cultures made in duplicate, $c .50 \%$ yielded growth in both bottles. The original 70 locally obtained blood samples yielded 12 isolates; these were derived from 12 of 80 cultures made from 50 samples of whole blood. As shown in table I, certain samples yielded two different isolates; in such instances, the two isolates were sometimes recognised simultaneously, but sometimes the second isolate was obtained from a duplicate culture, either simultaneously or subsequently. Two apparently identical isolates, obtained on different occasions from a single culture, were considered to represent a single isolation, and were recorded as such.

Isolates from cells and plasma. Eighteen isolates were obtained from 14 of 70 cultures of centrifuged blood cells, but none was obtained from 90 cultures inoculated with plasma separated in this laboratory, and only one was obtained from 55 samples of Italian plasma cultured in this laboratory. The infection thus appeared to be associated with the erythrocytes.

Other isolates. No bacterial rod forms other than BLE were found in the blood or cell-deposit cultures from normal subjects. One strain of Bacillus cereus occurred in plasma; three samples gave numerous Staphylococcus 
TABLE I

Periods from inoculation of cultures to first isolation of BLE from each subject

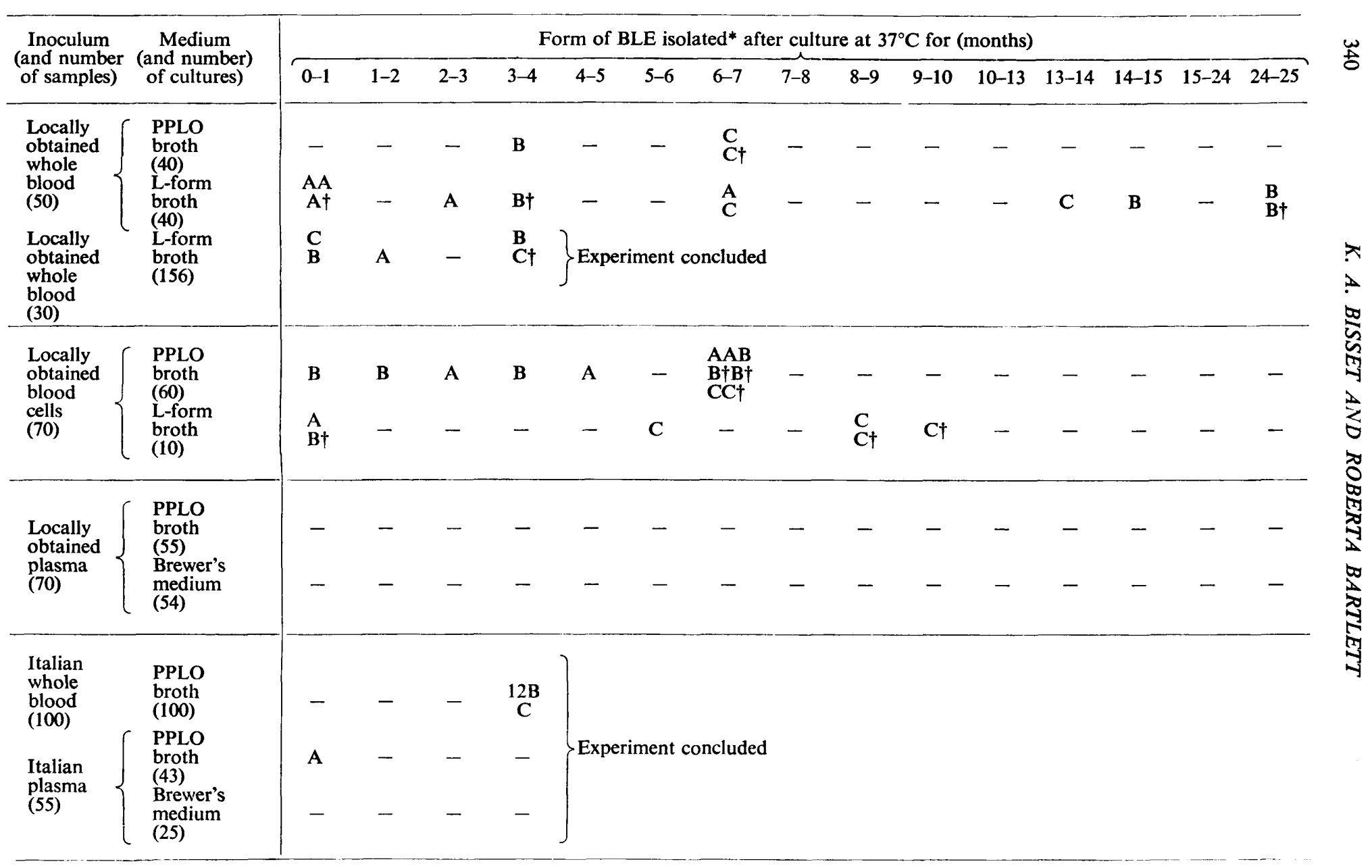

* $\mathrm{A}=$ Spheroplasts and very small rods; $\mathrm{B}=$ diphtheroid bacilli $\mathrm{C}=$ sporing bacilli; - = no isolate.

$\dagger \mathrm{A}$ second and distinct isolate from a single subject, as judged by cultural and morphological examination. 


\section{ERYTHROCYTE-ASSOCIATED BACTERIA}
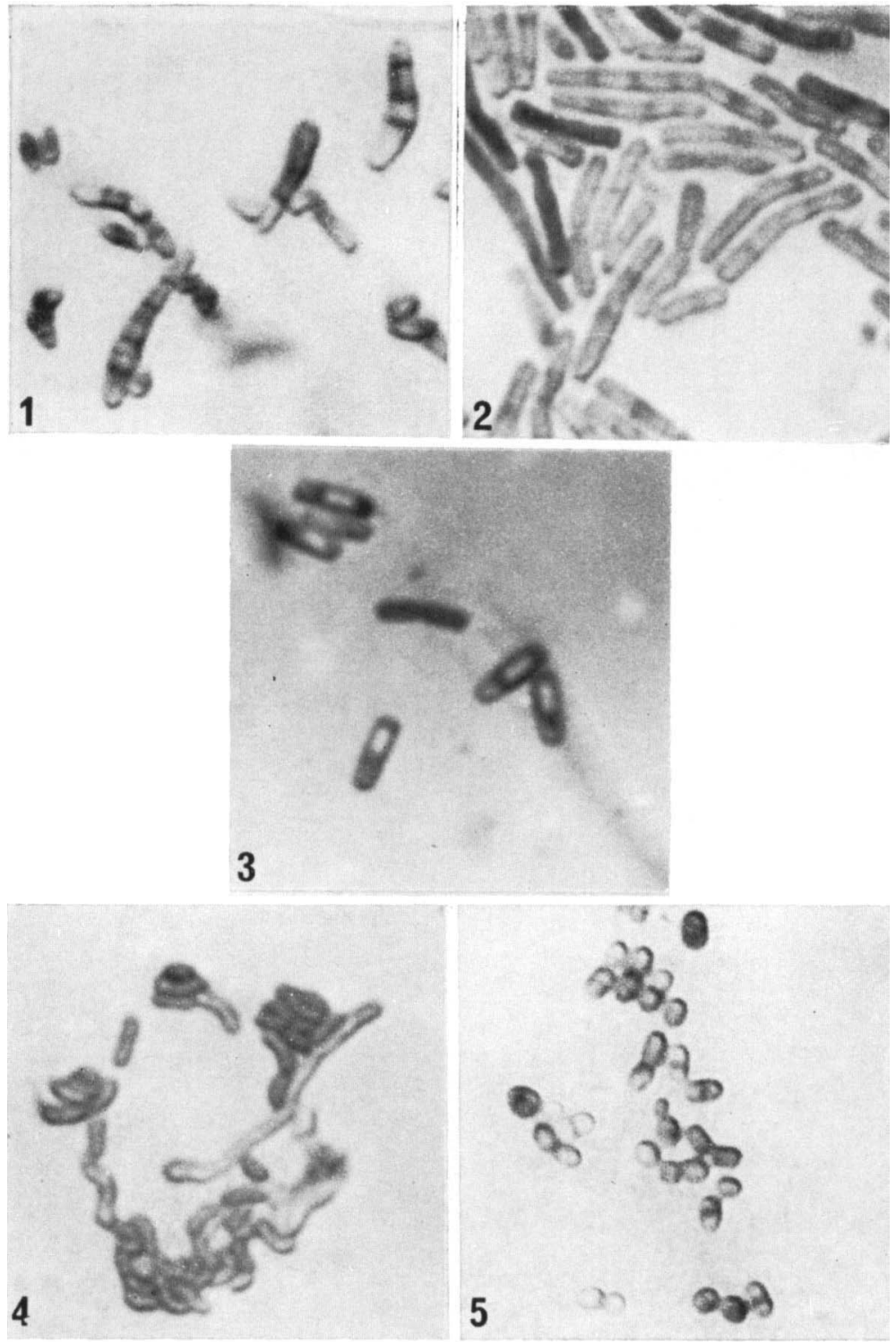

FIG. 1.-Typical diphtheroid bacillus, with corynebacterial morphology, occurring at all stages of culture. Phosphomolybdic acid and methyl green (PMG). $\times 2500$.

FIG. 2.-Rod morphologically resembling Bacillus licheniformis, but without endospores, arising from later stages of culture of the preceding form. PMG. $\times 2500$.

FIG. 3.--Sporogeneous, fully-reverted bacillus. PMG. $\times 2500$.

Fig. 4.--Soft-bodied, filamentous L-form type of diphtheroid. PMG. $\times 2500$.

FIG. 5.-Short, diphtheroid form, superficially resembling a coccus, but distinguishable by the parallel arrangement of the cross-walls. PMG. $\times 2500$. 
epidermidis after 24 hours' incubation, and six, including the plasma infected with $B$. cereus, yielded $S$. epidermidis after delays of up to several months. The delayed growth of $S$. epidermidis is considered to have indicated L-form infection (Tedeschi et al., 1976; Bisset, 1977), but the other incidents are thought to have been due to contamination. Nine of 100 whole-blood cultures taken in Ancona from hospital patients contained enterobacteria; eight contained staphylococci.

Morphology. Cultures, except unopened controls, were examined microscopically, both in the original fluid media and in subculture, at all stages of growth during the period of the experiment; acid-fast spheroplasts and small rods (Phase A, tables I and II) were isolated after incubation periods of up to c. 6 months, but especially between 2 and 4 months, and were slow to grow in subculture, taking 2-4 days to produce visible colonies. Diphtheroids (Phase B, tables I and II) were especially common at about 3 or 4 months after primary inoculation and grew well after 2-3 days in subculture (fig. 1). After 6 months, most isolates were in the morphological form of Bacillus licheniformis (Phase C, tables I and II) and grew overnight in subculture (fig. 2). Isolations ceased after 15 months, except that one primary culture yielded - after 25 months' incubation - the first isolate from the sample with which it had been inoculated. Endospores survived $80^{\circ} \mathrm{C}$ for at least $4 \mathrm{~h}$, and $100^{\circ} \mathrm{C}$ for a few minutes (fig. 3).

Several strains of diphtheroids produced occasional L-type filaments (fig. 4), and three when grown in fluid media were of short length and superficially resembled cocci (fig. 5), although a clear difference was revealed with cell-wall stains (Bisset, 1970).

Objects resembling spheroplasts were visible by microscopical examination,

TABLE II

Characters of BLE isolates and reversions

Morphology and staining

Origin (and number) of strains

Physiology

Phase A: spheroplasts

Tiny rods, highly pleomorphic; not always subcultivable; nonsporing; non-motile; usually acid-fast; Gram-negative, variable, or unevenly-staining

Phase B: diphtheroid forms

Non-sporing; non-motile; frequently acid-fast; Gram-positive, frequently stain in bands

Phase C: bacillus-like forms

Most sporing, with oval, subterminal spores; motile; non acid-fast; Gram-positive
New isolates (11)

S. Inoue (1)

D. H. Roberts (4)

G. Tedeschi (2)

New isolates (14)

P. Pease (1)

F. B. Siebert (3)

G. Tedeschi (7)

New isolates (12)

E. Alexander-

Jackson (4)

P. Pease (4)

G. Tedeschi (1)
Catalase positive; oxidase variable; physiologically inactive; fermentative metabolism or inert; relatively poor fermenters, giving regular positive reactions only with glucose and sucrose; frequently urease positive

Catalase positive; oxidase variable; fermentative metabolism; physiological activity variable, but positive reactions, where they occurred, were as for Bacillus-like forms

Catalase positive; oxidase positive; fermentative metabolism (see table III) 
in variable numbers, in $c .50 \%$ of blood or cell cultures after 1-2 weeks; many such cultures were macroscopically indistinguishable and it is recognised that some of the microscopical identifications of spheroplasts may have been unreliable.

The initial colonies of BLE spheroplasts were pale and very small, resembling mycoplasma colonies, but they became macroscopically visible after further incubation; those of diphtheroids, from later stages of culture, were larger, although very variable in size, and more opaque. Spheroplasts rapidly reverted to diphtheroids when subcultured on agar. Over a period of 8-10 weeks of growth in subculture, c. $70 \%$ of diphtheroids reverted further to non-sporing forms morphologically and culturally resembling Bacillus licheniformis; as mentioned elsewhere, this does not necessarily imply a further large increase in size. Sporogenous forms appeared after prolonged incubation of primary cultures, including those not previously opened; they could be detected microscopically, and produced numerous colonies on subculture.

Complete, direct reversions, to the fully sporogenous phase, were rather rare in pure subcultures of spheroplasts or diphtheroids, but could be induced to appear more rapidly by the method of Madoff (1974). This entailed growth in the presence of metabolic products derived from Staphylococcus epidermidis, either as an extract, or by diffusion from colonies, and will be the subject of a separate communication.

Physiological tests. Spheroplasts, and very small rods, whether isolated as such or derived from the spheroplasts, were inactive metabolically and, although capable of growing to a limited extent in peptone water or dilute serum, were inhibited by the concentrations of carbohydrates used in biochemical tests. Diphtheroids, whether isolated as such or derived from the foregoing forms, were metabolically rather weak by comparison with the later, more Bacillus-like stages, but tended to show a similar pattern of reactions (tables II and III). Eight strains were inhibited by carbohydrates in peptone-water sugars; they grew in serum-water sugars, but were capable of fermenting very few substrates and usually only after prolonged periods of incubation of up to 30 days. Glucose and sucrose were the sugars most regularly fermented. In parallel with their degree of apparent morphological reversion from spheroplasts to progressively larger and more actively growing, Gram-positive bacilli, the BLE isolates tended also to become more physiologically active in respect of hydrolytic abilities, the Voges-Proskauer reaction, and fermentation of carbohydrates. In all these respects, the spheroplasts and small, acid-fast bacilli were almost totally inactive; fully reverted bacilli were similar in their main characters to Bacillus licheniformis, but occasional deficiencies of various single physiological abilities occurred, as described by Pease (1970 and 1974), e.g., about $25 \%$ of strains failed to utilise citrate (table III). Diphtheroids and non-sporing bacilli were intermediate in their physiological properties, as in their morphology and colonial form. The results obtained with strains from other sources were similar (table II). Fully reverted strains were fairly consistent in their morphology, colonial appearance and physiology, and distinct from the strains of bacteria that could be isolated in the laboratories where this work was performed, and from the skin and respiratory passages of operators. 
TABLE III

Physiological characters of Bacillus licheniformis (system of Cowan and Steel, 1974) and fully reverted, sporing $B L E$

\begin{tabular}{l|cc}
\hline \multicolumn{1}{c|}{ Test } & $\overbrace{\begin{array}{c}\text { Bacillus } \\
\text { licheniformis }\end{array}}$ & sporing BLE $\dagger$ \\
& & \\
\hline Motility & + & 10 \\
Anaerobic growth in glucose broth & + & 10 \\
Citrate utilisation & + & 7 \\
Gelatin hydrolysis & + & 9 \\
Casein hydrolysis & + & 10 \\
Starch hydrolysis & + & 10 \\
Glucose fermentation (acid) & + & 10 \\
Arabinose fermentation (acid) & +- & 0 \\
Mannitol fermentation (acid) & +- & 7 \\
Indole production & - & 10 \\
Voges-Proskauer & + & 4 \\
Nitrate reduction & + & 10 \\
Urease & $\mathrm{d}$ & 0 \\
Catalase & + & \\
\hline
\end{tabular}

$*+=80-100 \% ;-=0-20 \% ; \mathrm{d}=21-79 \%$.

$\dagger$ Number positive out of 10 isolates tested.

Effect on erythrocytes in primary cultures. The blood or cell cultures from which the L-form of BLE could be isolated in subculture were either unchanged in appearance or developed a slightly darker brown colour, and occasionally a very slight pellicle formed on the glass at the surface of the fluid. In such cultures, diphtheroids could often be detected microscopically. Lysis appeared late, at 2-18 months after inoculation, and did not usually precede the first isolation of BLE.

\section{Controls}

Sampling procedure. The anticoagulant exposed at the blood-taking sessions gave negative results when cultured and incubated under the conditions used for the tests. Washings from the bottles and samples of the anticoagulant solution were proved by cultural methods to be sterile.

Environment. Exposed plates, swabs etc. from the laboratories and incubators in this department gave no cultures resembling BLE in the form in which it was usually isolated from blood. The level of contamination was exceedingly low, and on exposed plates only one colony of $B$. licheniformis appeared; 40 other colony types were obtained, of which 25 represented staphylococci and micrococci, and the remainder Bacillus cereus-mycoides, distinguishable by its much greater size, or B. lentus, having the same, small size as $B$. licheniformis but possessing a smooth colony and distinct physiological pattern.

Operators. Plates exposed in the vicinity of the operators gave results similar to those obtained from the environment (see above). Swabs cultured 
from the skin and respiratory passages of the operators and of other persons in the laboratory, over a period of 10 years, produced no organisms resembling BLE, except one colony of $B$. licheniformis from one person not associated with the experiment. It was rare to find any bacterial flora other than Gram-positive cocci on the dry skin; corynebacteria were occasionally isolated from mucous membranes, but these organisms were totally distinct in appearance and culture from the diphtheroid phase of BLE and, unlike the smaller forms of BLE, were never observed to be acid-fast.

Cultures of autoclaved and irradiated erythrocytes. The 90 culture controls that consisted of autoclaved mixtures of L-form broth and erythrocytes were incubated and subcultured at intervals of $c .1$ week for 12 weeks; more than 1000 subcultures were performed in the same laboratories as those used for the experiment, and by the same operators. All were negative.

The 60 culture controls that consisted of mixtures of L-form broth and irradiated erythrocytes were incubated and subcultured at intervals of 4 weeks for 6 months; 140 subcultures were made. All were negative.

These two negative control experiments, designed to confirm the efficacy of the aseptic precautions used for the subcultures in the original large-scale investigation, were performed subsequently to the latter. Accordingly, to provide simultaneous positive controls, cultures were made from 30 blood samples obtained from the original sources. Four samples were positive, yielding five cultures of BLE after c. 6 months' incubation; this resembled the earlier results (table I).

Media. The testing of media for sterility entailed a procedure that acted also as a control for culturing, subculturing and incubation; uninoculated bottles of media, incubated and subcultured at intervals (c. 350 subcultures) in parallel with the inoculated cultures throughout the entire experiment, remained completely sterile.

Parallel cultures. When a positive blood sample was cultured in different media, BLE was not invariably isolated from them all; this is attributable to the presence of only small numbers of BLE capable of reversion; however approximately $50 \%$ of positive samples yielded BLE in more than one culture. The corresponding figure based on pure chance would, with the time scale employed in this experiment, have been less than $5 \%$. BLE occurred in $30 \%$ of previously unopened primary cultures; this percentage was similar to that found in cultures opened repeatedly for subculture.

Effect of isolates on erythrocytes. Eight strains of BLE, of which two were fully reverted and six were in the diphtheroid phase, and four strains of saprophytic $B$. licheniformis, were inoculated into control cultures containing untreated blood. The reverted, sporogenous BLE and B. licheniformis lysed and destroyed the human or equine erythrocytes within $48 \mathrm{~h}$, producing a brown coloration. The diphtheroid strains were only slightly less actively lytic, taking from 2-10 days to produce the same effect. In primary cultures a similar effect was produced only after several months.

Indirect controls. Certain results, although not arising from procedures specifically designed to provide controls, merit recording. The isolation of 
BLE from human blood taken and cultured independently in Ancona, with different materials, methods and operators, serves as a positive control.

A negative control is provided by the almost total absence of BLE, or any other sporing organism, in cultures inoculated with plasma separated in this laboratory or in Italy.

A negative control of cultural procedures is provided by the concentration of isolations from cultures in the early months of incubation, and their absence between the 15th and 25th months in samples that had been regularly subcultured.

Controls are also provided by other projects that have been conducted in the same laboratories at the same time, some of which entailed incubation of a wide range of different media, including some of those used in the present experiments. In our work with actinomycetes, for example, cultures incubated for several months have rarely produced sporing bacilli of any kind as contaminants. Contamination, when it occurred, was usually produced by fungi or Grampositive cocci.

Independent diagnosis of species. An independent examination of 12 typical, sporogenous isolates identified in this laboratory as reverted BLE was made in the Microbiology Department of the West of Scotland Agricultural College through the kindness of Dr Selwyn Baines. The findings corresponded generally with those of this department (table III). The isolates did not completely correspond to any named species, being deficient in occasional physiological characters, as reported earlier in this paper and by Pease (1970 and 1974), but all fell into the subtilis-licheniformis group I; I; B, 1 (Breed, Murray and Smith, 1957). Nine of 12 were considered to be $B$. licheniformis although they varied in respect of citrate utilisation and the Voges-Proskauer reaction; the other three, although generally similar, were distinguished by their preferential aerobic growth. These aberrant physiological characters are within the range of variability of single strains of BLE in the course of reversion from the L-form.

\section{Discussion}

The present observations appear to demonstrate that the L-form of BLE, a member of the subtilis-licheniformis group of the Bacillaceae, closely resembling $B$. licheniformis, occurs in association with the blood and blood cells of normal human subjects, but not usually with the plasma. This association of bacillary L-forms with erythocytes has been illustrated in electronmicrographs by Pease (1970), Domingue, Schlegel and Woody (1976) and Tedeschi et al. (1978). About $30 \%$ of samples yielded isolates in cultures incubated for up to 15 months. This is in accordance with the results of Villequez (1965).

The infection may be universal in man, as claimed by Benedek (1955) and suggested by Pease (1974), but the organism's isolation and morphological reversion are delayed or inhibited in strains from healthy persons, so that its nature and very existence usually go unperceived. It is presumed that this inhibition is due to immunological factors, and may explain the apparent association of the infection with diseases having an autoimmune component (Pease, 1965a). 
The variable morphology of the reverting L-form of BLE has been a source of confusion. It may pass through stages resembling organisms of the genera Mycoplasma, Mycobacterium, Streptococcus, Corynebacterium, Listeria, Micrococcus or Bacillus, all of which have regularly been reported by different workers (see Pease, 1967a). Our evidence indicates that these are all stages in the reversion, and this serves to confirm the results of Alexander-Jackson (1954) and others (see Symposium, 1970), by whom the cycle has been described in detail, although not as originating from an L-form. Marmion (1976) criticised previous descriptions of reversions from spheroplast to diphtheroid, on the ground that serological evidence proving the relationship of the phases has been lacking: in fact, Pease (1965b and 1967b) produced such evidence for several similar reverted strains. However, the best proof of identity is the repeated derivation of each phase from the preceding; in this account we have presented evidence of such changes in several hundred pure cultures.

If this interpretation is contested, the alternative explanation is that our results were due to contamination of cultures, with a wide variety of organisms, but in regular progression mimicking the effects described. This seems unlikely as the reverted forms consistently belonged to the subtilis-licheniformis group of the genus Bacillus; they differed from all samples of contaminants found in these laboratories, and were identical regardless of their widely different sources. The intermediate forms were also highly characteristic.

Contamination could have occurred only during inoculation, incubation or subculturing, or in the taking of the blood samples. The BLE organism was found to occur in primary cultures that had not been opened previously, with the same frequency as in those from which subcultures had been made. Moreover, the occurrence of BLE in cultures of blood could not readily be explained in terms of contamination resulting from repeated subculturing unless the organism were found consistently in the environment. Thus we do not believe that the isolations of BLE were due to contamination, and we stress that numerous controls were included to show that contamination was not responsible.

Direct and indirect control data respectively were obtained from control experiments and from observations arising fortuitously in the course of other experiments. Negative results were obtained by the repeated subculturing of (1) uninoculated cultures, (2) inoculated but inadequately incubated cultures, (3) inoculated, autoclaved cultures, and (4) cultures inoculated with irradiated blood or with plasma; these four types of culture were subjected to a greater degree of handling than were the positive blood cultures. They also served as controls for the processes of inoculation and incubation. The negative cultures, incubations and subcultures totalled several thousands. On the other hand, the BLE organism grew and reverted well in cultures with cells, or plasma, or neither.

Containers of anticoagulant opened during the blood-sampling periods and subsequently sampled and cultured did not yield BLE, although left open for much longer than the test containers. The skin of human subjects has not been shown to carry BLE or any comparable species with a frequency likely to give rise regularly to a $30 \%$ contamination rate. Staphylococcus epidermidis, 
although it almost always occurs on human skin, was found at a very low frequency as a contaminant or as an L-form associated with the platelets (Tedeschi et al., 1976; Bisset, 1977).

The suggestion of Marmion (1976) that the diphtheroid phase is a common skin-dwelling corynebacterium cannot be substantiated by our evidence. The diphtheroid phase does not correspond physiologically to any species of Corynebacterium, according to the scheme of Cowan and Steel (1974); moreover, the distinctive, acid-fast forms, and the possession of spores by the reverted phase, put it unequivocally outside the genus Corynebacterium.

The skin of the donor is not sterilised by standard disinfecting procedures, and contamination could therefore have been derived from the skin; but if so, the organism so regularly encountered must surely be regarded as a highly unusual, erythrocyte-associated organism, and not as an ordinary contaminant. It is also apparent, from the experiment on the effect of reverted BLE upon erythrocytes in culture, that contamination with free-living BLE or B. licheniformis would not have produced the results observed, as the erythrocytes would rapidly have been lysed. Thus any such contaminant must have been an L-form, or have rapidly been transformed into an L-form, simply by growth in the presence of blood; however, the same observations show that BLE is not transformed in this way, and can grow or revert in simple media.

BLE organisms occur regularly in materials taken by different methods, from different sources, in different parts of the world; some such materials, e.g., excised tumours or synovial membranes (Symposium, 1970), are quite unlike the present blood samples, have no contact with skin, and yet are capable of providing many isolates of BLE. The present study throws no light on suggestions made in the past that BLE organisms play a part in the aetiology of cancer and arthritis, or are associated with immunological disturbance (Pease, 1965a; Symposium, 1976 and 1977).

\section{SUMMARY}

Thirty-eight strains of a Gram-positive bacterium identified as Bacillus licheniformis var. endoparasiticus (Benedek), referred to as BLE, were isolated in various stages of reversion from the L-forms, from 28 out of 100 samples of whole blood or erythrocytes from normal healthy subjects, after prolonged incubation. Similar results were obtained from 100 samples from hospital patients with conditions not usually associated with blood infection. BLE was isolated from only one of 125 samples of plasma, including those separated from infected erythrocytes.

Isolates from cultures incubated for up to 4 months were usually in the form of spheroplasts or diphtheroid bacilli; the fully reverted phase, resembling $B$. licheniformis, with the capacity to form endospores, was isolated occasionally from cultures aged 1-6 months, and it constituted about half the isolates recovered from cultures aged 6-25 months.

BLE was isolated in subculture, and with the usual frequency, in previously unopened, primary cultures. It did not occur in 1200 subcultures of 150 control cultures made with autoclaved or irradiated blood cells; it was not detected in 
the environment of the laboratory or blood-sampling areas, or on the skin or in the respiratory passages of the operators and other persons associated with the laboratory, where typical, saprophytic $B$. licheniformis was very rare.

It is concluded that this Bacillus species exists as an L-form, associated with the erythrocytes of a large proportion of normal persons, as previously recorded by several observers. Some of the morphological variants associated with the L-cycles have in the past been described as different organisms, for example Lforms of various bacteria or mycoplasmas, and the diphtheroid stage has been thought to belong to the genera Corynebacterium and Listeria. The sporogenous stage, although frequently described, has normally been discounted as a contaminant.

These observations do not admit of any conclusion in respect of the claims that such bacteria may have a role in arthritis, cancer or other diseases.

We wish to acknowledge the assistance given in this work by Ms Hilary Whitby and Ms Susan Balmer.

\section{Note}

Just after this paper was submitted a further piece of corroborative evidence was produced by Walker and RajBhandary (1978), in this University but entirely independently. These authors have shown that the tRNA of Mycoplasma mycoides subsp. capri is not only of a distinctively bacterial type but closely resembles that of Bacillus subtilis, and preliminary investigations suggest the same of other mycoplasmas. This information should be related to our conclusion that certain organisms that have consistently been identified as mycoplasmas, and specifically as Mycoplasma hominis by several authors, are unusually stable L-forms of a member of the genus Bacillus in the subtilis-licheniformis group.

\section{REFERENCES}

AleXANDER-JACKSON, E. 1954. A specific type of microorganism isolated from animal and human cancer: bacteriology of the organism. Growth, 18, 37.

BenedeK, T. 1955. Rheumatoid arthritis and psoriasis vulgaris, Ann Arbor, Michigan, p. 8.

Breed, R. S., Murray, E. G. D. AND Smith, N. R. 1957. Bergey's Manual of determinative bacteriology, 7 th ed., Baltimore, p. 614 .

BISSET, K. A. 1970. The cytology and life-history of bacteria, 3rd ed., Edinburgh, pp. 109 and 130.

BISSET, K. A. 1977. Isolations of micro-organisms from arthritic patients and healthy normals. Ann. rheum. Dis., 36, 96.

Bisset, K. A. AND STReEt, J. 1973. Morphological phases in the swarm of Bacillus licheniformis. J. gen. Microbiol., 76, 369.

Cowan, S. T. AND SteEL, K. J. 1974. Manual for the identification of medical bacteria, 2nd ed., Cambridge, p. 71.

Domingue, G. J., Schlegel, J. U. AND Woody, H. B. 1976. Naked bacteria in human blood. A novel concept for etiology of certain kidney diseases. Microbia, 2, (2), 3.

Duthie, J. J. R., Stewart, S. M., AleXander, W. R. M. AND Dayhoff, R. E. 1967. Isolation of diphtheroid organisms from rheumatoid synovial membrane and fluid. Lancet, 1, 142.

LIVINGSTON, V. W. AND ALEXANDER-JACKSON, E. 1970. A specific type of organism cultivated from malignancy: bacteriology and proposed classification. Ann. N.Y. Acad. Sci., 174, 636.

MADOFF, S. 1974. The influence of microbial factors on the reversion of L-forms of Haemophilus influenzae and streptococci. Abstr. A. Meeting Am. Soc. Microbiol., para G. 246, p. 61 . 
Markham, J. G. AND Myers, D. B. 1976. Preliminary observations on an isolate from synovial fluid of patients with rheumatoid arthritis. Ann. rheum. Dis., 35, 1.

Marmion, B. P. 1976. A microbiologist's view of investigative rheumatology. In Infection and immunology in the rheumatic diseases, edited by D. C. Dumonde, Oxford, p. 245.

Pease, P. E. 1965a. L-forms, episomes and autoimmune disease, Edinburgh.

PEASE, P. E. 1965b. The antigenic structure of PPLO (Mycoplasma hominis) and related bacteria. J. gen. Microbiol., 41, 299.

PEASE, P. E. $1967 a$. Tolerated infection with the sub-bacterial phase of Listeria. Nature, Lond., 215, 936.

PEASE, P. E. 1967b. Antigenic relationships between oral group D streptococci, some Haemophilus species and Mycoplasma hominis. J. gen. Microbiol., 49, 433.

PeASe, P. E. 1969. Bacterial L-forms in the blood and joint fluids of arthritic subjects. Ann. rheum. Dis., 28, 270.

PEASE, P. E. 1970. Morphological appearance of a bacterial L-form growing in association with the erythrocytes of arthritic subjects. Ann. rheum. Dis., 29, 439.

PEASE, P. E. 1974. Identification of bacteria from blood and joint fluids of human subjects as Bacillus licheniformis. Ann. rheum. Dis., 33, 67.

Pease, P. E., Nicholls, L. AND StUart, M. R. 1972. Evidence that precipitin cross-reactions between Listeria, Erysipelothrix and Bacillus licheniformis are not due to the Rantz antigen. J. gen. Microbiol., 73, 567.

RoBERTS, D. H. 1964. L-phase bacterial forms associated with infectious synovitis in chickens and turkeys. Res. vet. Sci., 5, 441.

SYMPOSIUM 1970. Unusual isolates from clinical material. Ann. N.Y. Acad. Sci., 174, 431.

SYMPOSIUM 1976. Infection and immunology in the rheumatic diseases, edited by D. C. Dumonde, Oxford, London, Edinburgh and Melbourne.

SYMPOSIUM 1977. Spheroplasts, protoplasts and L-forms of bacteria, edited by J. Roux, Institut National de la Santé et de la Recherche Scientifique, Colloque No. 64, Paris.

Tedeschi, G. G., Amici, D., Santarelli, I., Paparelli, M. and Vitali, C. 1976. Unstable L-forms of micrococci in human platelets. In Microbial ultrastructure: the use of the electron microscope, edited R. Fuller and D. W. Lovelock, Soc. Appl. Bact. Tech. Series 10, London, p. 325.

Tedeschi, G. G., Bondi, A., Paparelli, M. and Sprovieri, G. 1978. Electron microscopical evidence of the evolution of corynebacteria-like microorganisms within human erythrocytes. Experientia, $\mathbf{3 4}, 458$.

VILlEQUEZ, E. 1965 . L'étude biochimique du sang ne doit pas méconnaître le rôle des évolutions de corpuscles existant dans ce milieu. Proc. 10th Congr. Int. Soc. Blood Transf., Stockholm, Bibliotheca Haematologia, Basel, vol. 23, p. 752.

WALKER, R. T. AND RAJBHANDARY, U. L. 1978. The nucleotide sequence of formylmethionine tRNA from Mycoplasma mycoides sp. capri. Nucleic Acids Res., 5, 57. 